\title{
Bragg Grating Waveguide Array Ultrafast Laser Inscribed into the Cladding of a Flat Fiber
}

\author{
Stephen J. Beecher, Robert R. Thomson, Graeme Brown, Andrew. S. Webb, Jayanta. K. Sahu and Ajoy, K. \\ Kar \\ School of Engineering and Physical Sciences, Heriot-Watt University, Edinburgh, EH14 4AS, Scotland, UK \\ Optoelectronics Research Centre, University of Southampton, Southampton SO17 1BJ, United Kingdom \\ Author e-mail address: R.R.Thomson@hw.ac.uk
}

\begin{abstract}
We report the fabrication and initial characterization of a waveguide sensor array in the cladding of a flat fiber. The sensor, designed to independently measure the strain on three Bragg grating waveguides, exploits the true three dimensional fabrication technology of ultrafast laser inscription by placing these gratings in a non-planar configuration.
\end{abstract}

\section{Introduction}

Fiber Bragg gratings are among the most heavily utilized fiber sensing elements and have for many applications displaced their electrical counterpart, the strain gauge [1]. The geometry of the fiber, however, places constrains on the recoverable information to one dimension, parallel to the fiber core, yielding no information about loading direction.

Non-linear absorption at the focus of an ultrashort pulse in a material can result in structural changes, which under the correct conditions, can lead to a localized increase in the refractive index of the material. By translating the substrate through the laser focus this localized refractive index increase can be extended into a channel waveguide [2]. By modulation of the inscription laser during translation a longitudinal variation in the refractive index profile can be achieved, this can be used to create waveguide Bragg gratings [3]. Recently this technique has been extended to multiscan waveguides, waveguides with cross sections built up of multiple scans of the substrate through the laser focus [4].

In this paper we report the fabrication of three waveguides meeting in a $3 \times 3 \mathrm{Y}$-splitter, fanning out to move the waveguides out of a plane before a straight parallel Bragg grating section to each waveguide. This non-planar geometry should allow for differential strain measurement and therefore direction and curvature of the device to be inferred.

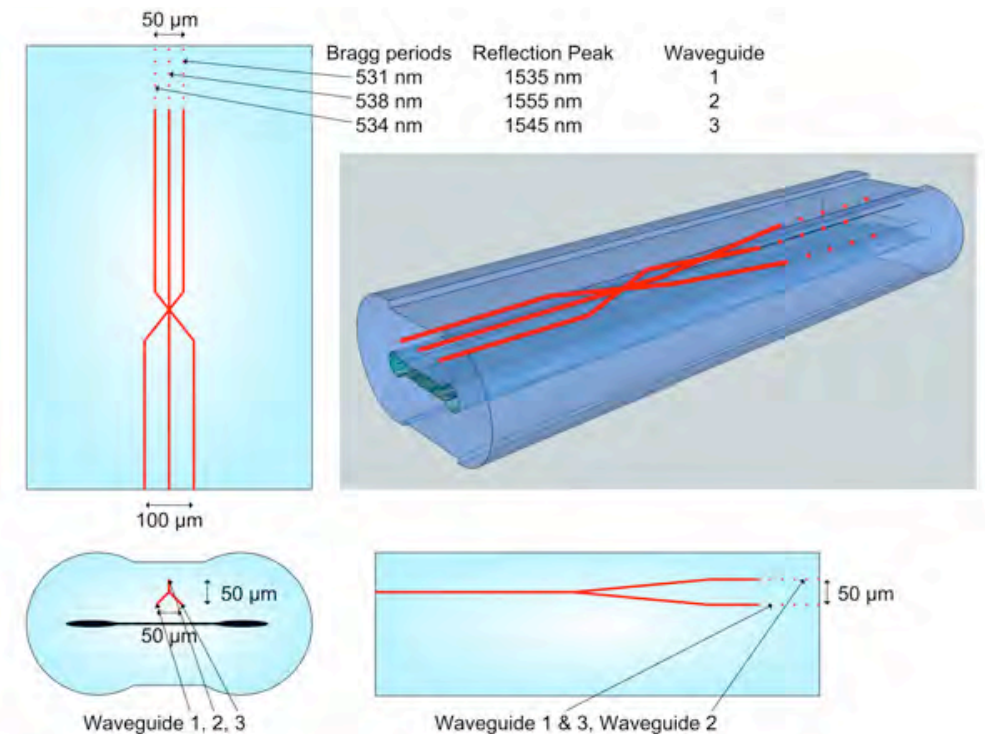

Figure 1. Schematic of the device. An in-plane $3 \times 3$ Y-splitter followed by a fan-out section to move the waveguides out-of-plane, each waveguide contains a Bragg grating section of a different period. 


\section{Device design}

This work was undertaken using an experimental substrate obtained from the Optoelectronics Research Centre (ORC), at the University of Southampton. The substrate used is a 'flat fibre', which aims to apply the mass production technology of optical fibers to the production of substrates for UV laser writing of planar lightwave circuits, more complete information on the 'flat fiber' can be found in Webb et al. [5]. Inscription equipment and parameter are identical to those optimized for fused silica and reported by Beecher et al. [6]. The device design is shown in Figure 1.

\section{Characterization}

A fiber was bonded to a v-groove, aligned to the central waveguide on the input and bonded to the 'flat fiber'. A fiber circulator was used to couple light from an erbium doped fiber amplified spontaneous emission (ASE) source into the waveguide with the reflected light sent to an optical spectrum analyzer (OSA). After bonding, the device was placed in a temperature controlled oven and the spectrum of the reflected light was measured between $40{ }^{\circ} \mathrm{C}$ and $75{ }^{\circ} \mathrm{C}$. The temperature response of each of the three Bragg waveguides sections was found to be linear over the investigated temperature range with the three cores displaying temperature sensitivities of $10.5,12.2$ and $10.1 \mathrm{pm} /$ ${ }^{\circ} \mathrm{C}$ respectively. The significant difference in the gradient associated with the grating closest to the outside of the fiber is thought to be due to differential thermal expansion between the heavily Ge doped core of the 'flat fiber', this strains the cladding of the 'flat fiber' as it is heated resulting in a different temperature sensitivity for waveguides a different distance away from this core.
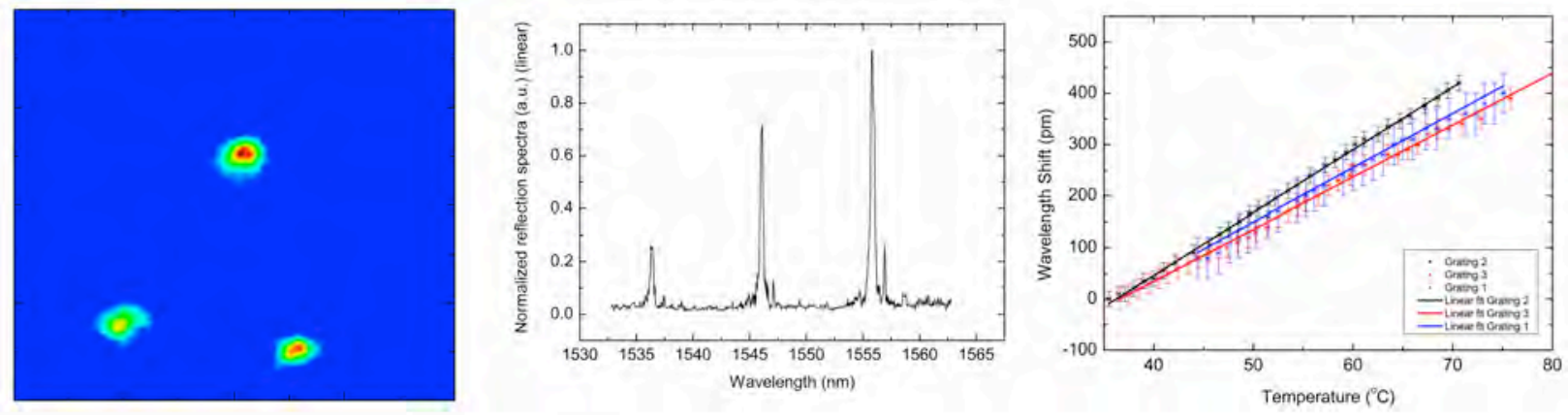

Figure 2. Device characteristics. Left: Near field image of the flat fiber end facet when coupling light into the central input waveguide. Middle: the spectra measured by the OSA for coupling into the central waveguide. Right: the shift in the reflection peak of each grating as temperature is varied.

\section{Conclusions}

We have demonstrated a complex three dimensional device fabricated into the cladding of a flat fiber. The device shows promise for strain sensing in applications where the recovery of directional information is necessary, potentially replacing arrays of fiber Bragg gratings with a single compact device. Temperature sensitivity for the three Bragg gratings is presented and future work will focus on the performance of this device in strain sensing applications.

\section{References}

[1] B. Culshaw and A. Kersey, "Fiber-Optic Sensing: A Historical Perspective," J. Lightwave Technol. 26, 1064-1078 (2008).

[2] K. M. Davis, K. Miura, N. Sugimoto, and K. Hirao, "Writing waveguides in glass with a femtosecond laser," Opt. Lett. 21, 1729-1731 (1996).

[3] H. Zhang, S. M. Eaton, and P. R. Herman, "Single-step writing of Bragg grating waveguides in fused silica with an externally modulated femtosecond fiber laser," Opt. Lett. 32, 2559-2561 (2007).

[4] G. Brown, R. R. Thomson, A. K. Kar, N. D. Psaila, and H. T. Bookey, "Ultrafast laser inscription of Bragg-grating waveguides using the multiscan technique," Opt. Lett. 37, 491-493 (2012).

[5] A. S. Webb, E. M. Adikan, J. K. Sahu, R. J. Standish, C. B. E. Gawith, J. C. Gates, P. G. R. Smith, and D. N. Payne, "MCVD planar substrates for UV-written waveguide devices," Electron. Lett. 43, 517-519 (2007).

[6] S. J. Beecher, R. R. Thomson, B. P. Pal, and A. K. Kar, "Single Stage Ultrafast Laser Inscription of a Side-Polished Fiber-Like Waveguide Sensor," Sensors Journal, IEEE 12, 1263-1266 (2012). 